\title{
Usual intake modelling is essential in assessing nutritional adequacy in populations
}

\section{Abstract}

Using short-term assessment methods to study diet provide too wide population intake distributions leading to overestimation of tail probabilities. Usual intake modelling reduces the variation in the distribution. The objective of this study was to evaluate the amount of bias in proportions of population under average requirement (AR) or recommended intake (RI) using the mean intake of two days compared to usual intake distribution. The FinDiet 2017 Survey was carried out in a nationally representative sample from the population register including adults aged 18-74 years. The data included two non-consecutive 24-hour dietary recalls from 1655 participants (response rate 53\%). Weighing factors were used to correct for non-participation bias. Usual intake was estimated using statistical program SPADE (RIVM, The Netherlands). The proportions of the population below AR or RI (when AR is not available) according to NNR 2012 were evaluated using two days mean intake and the usual intake distribution. These were further compared to evaluate the bias in two days mean intake. The bias associated with two days mean intakes was substantial in some of the nutrients. For example, for vitamin $\mathrm{C}$ and $\mathrm{D}$ intakes the difference between the proportions using two days mean and usual intake was over 10 percentage points. For vitamin C intake from food the proportions below AR ( $60 \mathrm{mg} / \mathrm{day}$ for men, $50 \mathrm{mg} / \mathrm{day}$ for women) were $38 \%$ for men and $20 \%$ for women using two days mean but 13 percentage points smaller for both sexes based on usual intake. The proportions below AR $(7.5 \mu \mathrm{g} /$ day) for vitamin D from food were $25 \%$ for men and $40 \%$ for women using two days mean and $14 \%$ for men and $30 \%$ for women using usual intake. When taking the intake from supplements into account, the proportions for vitamin D intake were $16 \%$ for men and $21 \%$ for women based on two days mean but only $7 \%$ for both sexes using usual intake. For calcium intake from food the proportion below AR (500 mg/day) was small (7\% for men and $8 \%$ for women) using two days mean but more than $50 \%$ smaller using usual intake (3\% for both sexes). Using two days mean intake to estimate nutritional adequacy can lead to considerable biased results due to extraneous day-to-day variation in the intake distribution. Therefore, usual intake modelling should always be applied to intakes derived from short-term dietary assessment methods when evaluating nutritional adequacy.

\section{Conflict of Interest}

There is no conflict of interest 